\title{
Großsprech: Vorreiter und Vorreiterrolle
}

Liebe Leserinnen und Leser

im vorläufigen Koalitionsvertrag vom 07. Februar 2018 ist zehn Mal davon die Rede, dass Deutschland mit dem Anspruch einer Vorreiterrolle nicht nur sich selbst, sondern auch Europa und die Welt erstaunen lassen will. Abgesehen davon, dass diese Rolle hinsichtlich der Wirkungen nicht definiert wird, sind die Bereiche (hier in der Reihenfolge ihrer Nennung im Text) in denen sie postuliert wird gewaltig:

- Klimaschutz EU

- Gigabit-Netze in alle Regionen

- FinTech

- Tierwohl

- Klimaschutz Klimaziele

- Faire EU-Handelspolitik

- Digitale Innovationen im Gesundheitswesen

- Energiewende

- Open-Data

- Faire Handelspolitik mit Afrika

Nüchterner geht es dagegen im Kapitel Digitalisierung zu. Hier spürt man die Schatten der Vergangenheit: Deutschland hatte ja über einen längeren Zeitraum technologisch motivierte Entwicklungen - insbesondere mit Bezug zur IT-Sicherheit - vorangetrieben, ohne vorrangig auf europäische und internationale Kooperation zu setzen. Sie konnten nur rudimentär realisiert werden und haben dem Ansehen deutscher Konzepte eher geschadet. Allerdings findet man auch in diesem Kapitel die Vision „Deutschland zu einem weltweit führenden Standort bei der Erforschung von künstlicher Intelligenz" zu entwickeln.

So lassen sich die Ansprüche vielleicht populär beschreiben:

- Der Vorreiter bildet die Spitze einer Kompetenzgemeinschaft, die Übrigen folgen freiwillig - aber geordnet?

- Bei der Vorreiterrolle ist der Anspruch auf,Führung' implizit gegeben. Für Andere ist nur Erfolg zu erwarten, wenn sie folgen.

Seit Jahren machen derartige Ansprüche den Fachleuten vieler Sparten Schwierigkeiten. Sie kennen natürlich die globalen Aktivitäten, deren Erfolge oder Misserfolge, die wissenschaftlichen Ressourcen und die international führenden Experten und können deshalb die deutschen Ansprüche nicht vorbehaltlos unterstützen. Häufiger haben Sie auch schon erlebt, dass frühere Meldungen dieser Art auf globalem Parkett - weil sie sich als nicht realisierbar erwiesen haben - verspottet wurden. Ein peinliches Statement lautet dann: Ihr deutschen Fachleute habt die Politik schlecht beraten oder mit falschem Opportunismus solche Zielstellungen ertragen.

Es ist deshalb nicht verwunderlich, wenn ein Koalitionsvertrag zwischen Union und SPD mit politischen Vorgaben für die Regierungsarbeit in dieser Legislaturperiode hinsichtlich derartiger Ziele als unverhältnismäßig empfunden wird. Stattdessen sollte die Politik eine fachlich begründete Bewertung der deutschen Ressourcen für die Digitalisierung im gesellschaftlichen Kontext vornehmen. Ein proportional plausibler Ausbau dieser Ressourcen sollte für erfolgsträchtige Bereiche als Machbarkeitsvoraussetzung umgesetzt werden. Es wäre auch nichts dagegen einzuwenden, wenn der notwendige europäische und globale Rahmen - beispielsweise für Standardisierung und Marktzugang - definitiv als notwendige Voraussetzung für die Entwicklung eines Wettbewerbs um das Gewicht deutscher Kompetenz in den genannten Bereichen konsequent das politische Handeln einer neuerlichen GroKo bestimmen würde.

Mit herzlichen Grüßen, Ihr

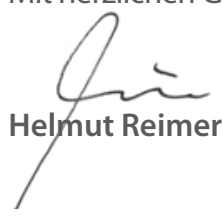

